\title{
Resveratrol induces human keratinocyte damage via the activation of class III histone deacetylase, Sirt1
}

\author{
JU-HEE LEE, JIN-SHANG KIM, SANG-YOUEL PARK and YOU-JIN LEE \\ Biosafety Research Institute, Department of Biochemistry, College of Veterinary Medicine, \\ Chonbuk National University, Iksan, Jeonbuk 54596, Republic of Korea
}

Received June 24, 2015; Accepted August 6, 2015

DOI: $10.3892 /$ or.2015.4332

\begin{abstract}
Human skin diseases are various and induce chronic inflammatory disorders, including psoriasis, atopic dermatitis and certain forms of ichthyosis. Psoriasis is a chronic inflammatory skin disease characterized by circumscribed, red, thickened plaques. Regulation of the balance between growth, differentiation and death is critical to keratinocytes; when altered, epidermal keratinocytes undergo hyperproliferation, abnormal differentiation and inflammatory infiltration. In the present study, we focused on the effects of resveratrol, found in red wine and peanuts, on the cell death of keratinocytes. We additionally studied the mechanism of resveratrol on Sirt1, a class III histone deacetylase, and Akt phosphorylation. Resveratrol caused apoptosis and increased Sirt1 expression in human HaCaT keratinocytes, following a decrease in the p62 protein level. Inhibition of Sirt1 by Sirt1 inhibitor restored cell viability and protein levels. Furthermore, we showed that resveratrol-induced Sirt1 blocked Akt phosphorylation. The present results indicated that resveratrol inhibited the Akt pathways by inducing Sirt1, thus leading to cell death. These data suggest that resveratrol-mediated activation of Sirt1 histone deacetylase may be a potential therapeutic target for skin diseases including psoriasis.
\end{abstract}

\section{Introduction}

Psoriasis is a common chronic skin disease that is characterized by hyperproliferation and disrupted differentiation of keratinocytes (1-3). Psoriasis leads to a decrease in apoptotic cell death in keratinocytes and an increased resistance of intra-lesional keratinocytes to apoptosis (4). We studied the inhibition of keratinocyte proliferation and the regulation of keratinocyte differentiation by induced apoptosis for the treatment of psoriasis.

Correspondence to: Dr You-Jin Lee, Biosafety Research Institute, College of Veterinary Medicine, Chonbuk National University, 79 Gobongro, Iksan, Jeonbuk 54596, Republic of Korea

E-mail: glocalvet@gmail.com

Key words: resveratrol, Sirt1, keratinocytes, apoptosis
Resveratrol (3,5,4'-trihydroxy-trans-stilbene) is a natural polyphenol, mainly found in grapes, berries and red wine (5). There are several studies on the bioactivities of resveratrol including anti-inflammatory, antioxidant, antimicrobial and neuroprotective effects (6).

Additionally, resveratrol has strong chemopreventive effects against skin, breast, prostate and lung tumors (7). The cancer preventive effects of resveratrol were demonstrated in the prevention of tumor growth in an animal model (8). Resveratrol is reportedly an activator of Sirtl both in vivo and in vitro $(9,10)$.

Silent information regulator 2 (Sir2) homolog 1 (Sirt1) is a member of the conserved family of NAD-dependent deacetylases, the sirtuin family, that function in enzymatic cleavage of NAD to the deacetylation of protein substrates. Sirtl plays a critical role in the regulation of numerous cellular processes, including cell cycle progression, nutrient metabolism, cellular ageing (11) and cell apoptosis (12). Sirt1 has nuclear substrates such as p53, and the DNA repair proteins APE/Ref1 and PARP1 (13-15). Resveratrol was found to inhibit cell proliferation and differentiation by increasing the expression of Sirt1 mRNA (16).

The serine/threonine kinase Akt, also known as protein kinase $\mathrm{B}$ (PKB), is a downstream effector of phosphatidylinositol 3-kinase (PI3K), activated by several stimuli, such as growth factor stimulation, stress or protein phosphatase inhibitors, in a PI3K-dependent manner (17). AKT is involved in multiple cellular signaling pathways and functions as a transducer of many actions initiated by growth factors and other receptors that activate PI3K (18-20). One of the major roles of Akt is to regulate cell survival $(18,21)$, including apoptosis and tumorigenesis as an oncogene (22). Recent studies have shown that Sirt1 inactivates Akt and suppresses survivin expression and the subsequently activated mitochondrion-mediated pathway, including MMP activity, cytochrome $c$ release and caspase activation (23).

The novel finding of the present study was that resveratrol induced HaCaT keratinocyte cell death. We investigated the role of resveratrol on the apoptosis of human $\mathrm{HaCaT}$ keratinocytes and clarified the mechanisms. Resveratrol was associated with increased expression and activation of Sirt1, by enhancing the deacetylation of $\mathrm{p} 53$, which downregulated Akt phosphorylation. The results indicated that resveratrol-induced HaCaT keratinocyte cell death may be regulated by a Sirt1/phospho-Akt-dependent signaling pathway. 


\section{Materials and methods}

Cell culture. HaCaT, an immortalized, non-tumorigenic human keratinocyte cell line was maintained in Dulbecco's modified Eagle's medium (DMEM) supplemented with $10 \%$ fetal bovine serum (FBS) and $1 \%$ antibiotics under standard conditions $\left(37^{\circ} \mathrm{C}, 5 \% \mathrm{CO}_{2}\right.$, in a humidified incubator). $\mathrm{HaCaT}$ cells were seeded in $60-\mathrm{mm}$ culture dishes in standard medium, or in the presence of different concentrations of resveratrol (25 and $100 \mu \mathrm{M}$ ) for the proliferation studies. Triplicate dishes were trypsinized at appropriate intervals, and the cell number was determined by counting cell suspension in a Neubauer hemacytometer. The values reported represent the mean \pm SEM of 3 independent samples per each experimental point.

Crystal violet assay. Cell viability was evaluated by crystal violet staining. Briefly, the cells were stained with staining solution $(0.5 \%$ crystal violet in $30 \%$ ethanol and $3 \%$ formaldehyde) for $10 \mathrm{~min}$ at room temperature (RT) and washed 4 times with water. The stained cells were lysed with $1 \%$ sodium dodecyl sulfate (SDS), and the absorbance was measured at $550 \mathrm{~nm}$. Cell viability was calculated based on the relative dye intensity compared with the controls.

Lactate dehydrogenase (LDH) assay. Cytotoxicity was assessed in the supernatants using the LDH cytotoxicity detection kit (Takara Bio, Tokyo, Japan) according to the manufacturer's protocol. LDH activity was determined by measuring the absorbance at $490 \mathrm{~nm}$ using a SpectraMax M2 microplate reader (Molecular Devices, Sunnyvale, CA, USA).

Annexin V assay. Apoptosis was assessed in detached cells with an Annexin V assay kit (Santa Cruz Biotechnology, Santa Cruz, CA, USA), according to the manufacturer's protocol. Annexin $\mathrm{V}$ levels were determined by measuring fluorescence at a 488-nm excitation and a 525/30 emission using the Guava easyCyte HT System (Millipore, Bedford, MA, USA).

Western blot analysis. HaCaT cells were lysed in buffer [25 mM HEPES, $100 \mathrm{mM} \mathrm{NaCl}, 1 \mathrm{mM}$ EDTA, $5 \mathrm{mM} \mathrm{MgCl}$, $0.1 \mathrm{mM}$ DTT (dithiothreitol) and a protease inhibitor mixture at $\mathrm{pH}$ 7.4]. Proteins were electrophoretically resolved by $10-15 \%$ sodium dodecyl sulfate polyacrylamide gel electrophoresis and transferred to a nitrocellulose membrane. Immunoreactivity was detected through sequential incubations with horseradish peroxidase-conjugated secondary antibodies and enhanced chemiluminescence reagents. The antibodies used for immunoblotting were Sirt1 (Santa Cruz Biotechnology), phospho-Akt, acetyl-p53 (both from Epitomics, Burlingame, CA, USA) and $\beta$-actin (Sigma-Aldrich, St. Louis, MO, USA). Images were examined using a Fusion-FX7 imaging system (Vilber Lourmat, Marne-la-Vallée, France).

Construction of recombinant adenoviruses. The Sirt1overexpressing adenovirus (Ad-Sirt1) was provided by Professor Byung-Hyun Park of Chonbuk National University (Jeonju, Jeonbuk, Korea). The lacZ-bearing adenovirus (Ad-lacZ) was used as the control. Recombinant adenoviruses were amplified in human embryonic kidney (HEK)-293 cells and purified using the Vivapure AdenoPACK kit (Sartorius AG, Göttingen, Germany) according to the manufacturer's instructions.

Statistical evaluation. All data are expressed as mean \pm standard deviation and were compared by Student's t-test, analysis of variance and Duncan's test using SAS statistical software version 9.1 (SAS Institute, Cary, NC, USA). Results were considered significant at $\mathrm{p}<0.05, \mathrm{p}<0.001$ or $\mathrm{p}<0.01$, as appropriate.

\section{Results}

Resveratrol induces cell damage in HaCaT keratinocytes. We first examined the influence of resveratrol on the cell viability of human $\mathrm{HaCaT}$ cells to determine whether resveratrol induces cell death or promotes cell proliferation and cell survival. Cells were exposed to different concentrations $(5,10,20$ and $50 \mu \mathrm{M})$ of resveratrol for $12 \mathrm{~h}$. After resveratrol treatment, based on the cell morphology and the number of cells by microscopy and crystal violet assay, we determined that resveratrol induced cell death (Fig. 1A and B). We next performed the LDH assay for detecting cell death. As shown in Fig. 1C, resveratrol treatment significantly increased LDH release. The Annexin V-positive cell population was also increased following resveratrol treatment, compared to that of the control (Fig. 1D). These data indicated that resveratrol induced $\mathrm{HaCaT}$ cell death.

Resveratrol induces cell death through the Sirt1 pathway. We examined the Sirtl protein levels in the HaCaT keratinocytes treated with resveratrol to understand the molecular mechanism of resveratrol in human keratinocytes. Resveratrol increases the deacetylase activities of Sirt1. Hence, we investigated whether resveratrol induced cell death by increasing Sirt1. First, we evaluated Sirt1 expression following resveratrol treatment at concentrations of 5, 10, 20 and $50 \mu \mathrm{M}$. Sirt1 protein levels dose-dependently increased in the resveratrol-treated group as compared to the control group (Fig. 2A). We next determined whether resveratrol-induced Sirt1 had deacetylase activity by measuring the acetylated $\mathrm{p} 53$ protein levels. Acetyl-p53 protein levels were gradually decreased (Fig. 2A). Moreover, sirtinol, Sirt1 inhibitor, inhibited the resveratrol-induced increase in Sirt1 protein and decrease in acetyl-p53 protein (Fig. 2B).

Next, to determine whether resveratrol-induced Sirt1 activation is related to cell death, $\mathrm{HaCaT}$ keratinocytes were treated with resveratrol with or without sirtinol. Crystal violet assay showed that resveratrol induced cell death and sirtinol restored cell viability (Fig. 2C). Consistent with these results, resveratrol-treated cells exhibited increased LDH release, while treatment with sirtinol blocked the effects of resveratrol (Fig. 2D). The influence of sirtinol on resveratrol-induced apoptosis in HaCaT keratinocytes was determined by the Annexin V assay (Fig. 2E). The resveratrol-mediated increase in Annexin V-positive cells was decreased by treatment with sirtinol. These results demonstrated that resveratrol induced cell death via Sirt1.

Resveratrol-mediated cell death is associated with Akt phosphorylation. The effect of resveratrol on apoptotic proteins 


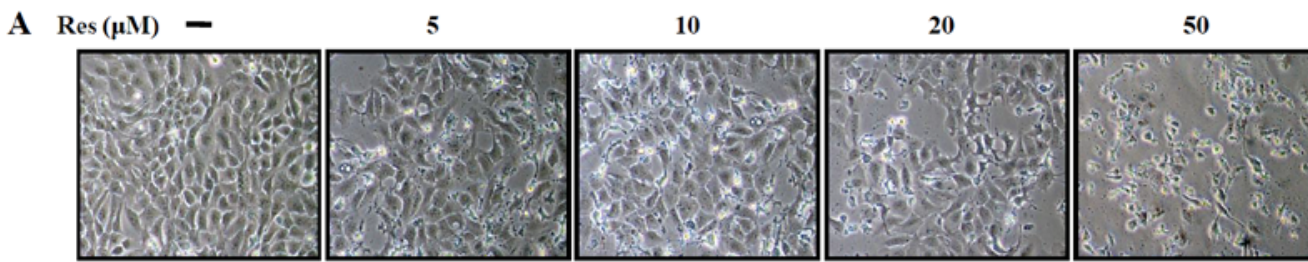

B

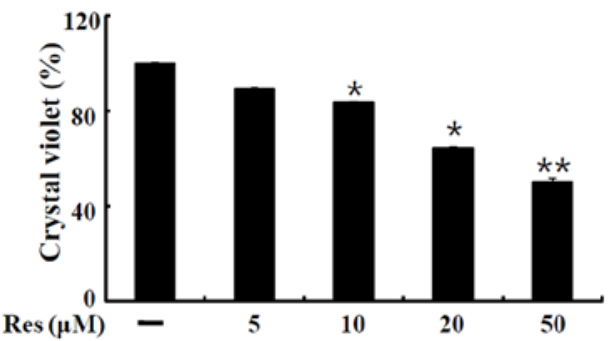

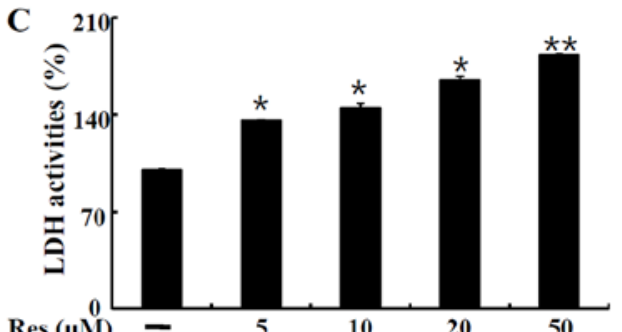

$\operatorname{Res}(\mu \mathrm{M})-$

D

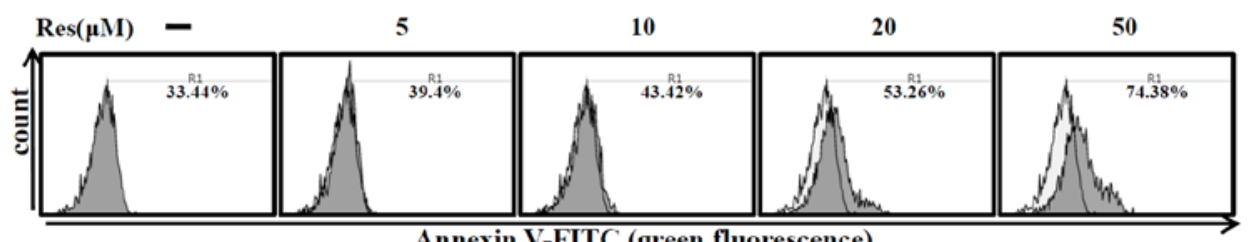

Annexin V-FITC (green fluorescence)

Figure 1. Resveratrol causes cell death in $\mathrm{HaCaT}$ keratinocytes. HaCaT keratinocytes were treated with resveratrol at increasing concentrations $(5,10,20$ and $50 \mu \mathrm{M}$ ) for $12 \mathrm{~h}$. (A) The treated cells were photographed using light microscopy. (B) Cell viability was measured by crystal violet staining. Viability of the control cells was set at $100 \%$, and viability was measured relative to the control. Bar graph indicates the mean \pm SEM $(n=3)$. (C) Lactate dehydrogenase (LDH) assay was used to quantify LDH release into the medium. Bar graph indicates the mean $\pm \mathrm{SEM}(\mathrm{n}=3) .{ }^{*} \mathrm{p}<0.05,{ }^{* *} \mathrm{p}<0.01$, significant differences between the control and each treatment group. (D) Cell viability was measured by the Annexin V assay. Res, resveratrol.

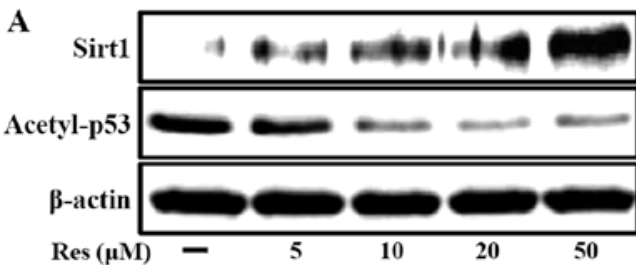

C

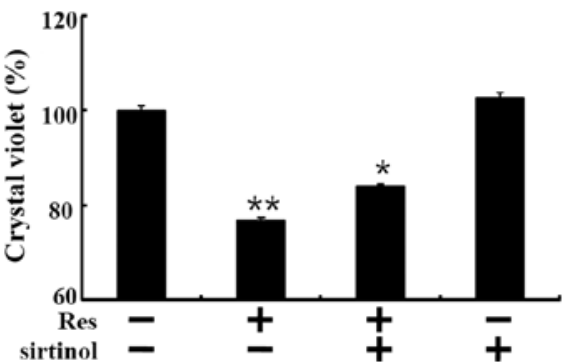

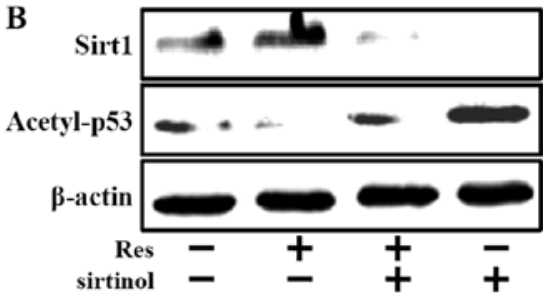

D
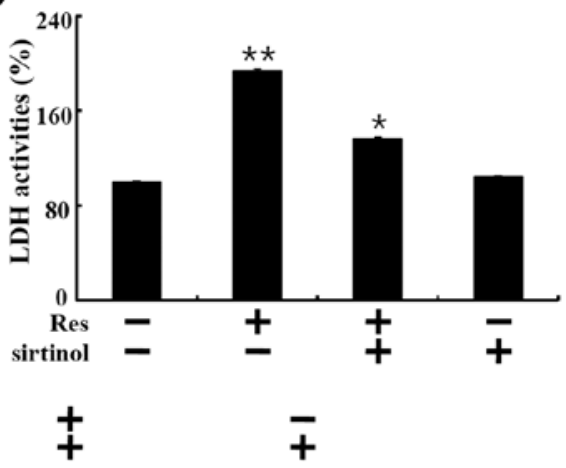

$\overline{+}$

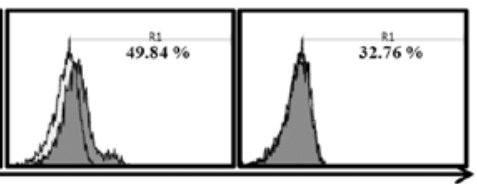

(green fluorescence)

Figure 2. Resveratrol regulates Sirt1 and acetyl-p53 protein levels in HaCaT keratinocytes. (A) HaCaT keratinocytes were incubated with the indicated concentrations of resveratrol $(5,10,20$ and $50 \mu \mathrm{M})$ for $12 \mathrm{~h}$. The treated cells were assessed for Sirt1 and acetyl-p53 by western blot analysis. Results were normalized to $\beta$-actin. (B) HaCaT keratinocytes were treated with $50 \mu \mathrm{M}$ of resveratrol in the presence of Sirtl inhibitor (sirtinol) for $12 \mathrm{~h}$. The treated cells were assessed for Sirt1 and acetyl-p53 by western blot analysis. (C) Cell viability was measured by crystal violet staining. Viability of the control cells was set at $100 \%$ and viability relative to the control was measured. (D) LDH assay was used to quantify LDH release into the medium. Bar graphs indicate the mean $\pm \operatorname{SEM}(\mathrm{n}=3) .{ }^{*} \mathrm{p}<0.05,{ }^{* * *} \mathrm{p}<0.01$, significant differences between the control and each treatment group. (E) Cell viability was measured by the Annexin $\mathrm{V}$ assay. Res, resveratrol. 
A

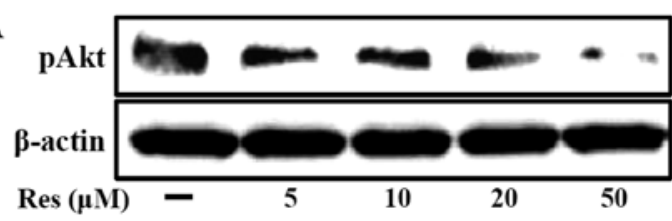

C

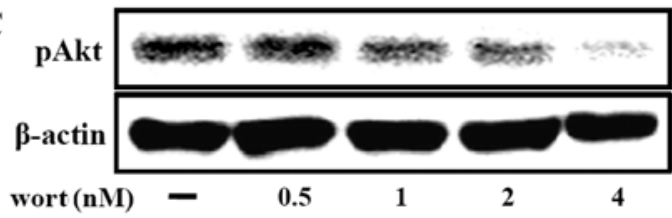

B
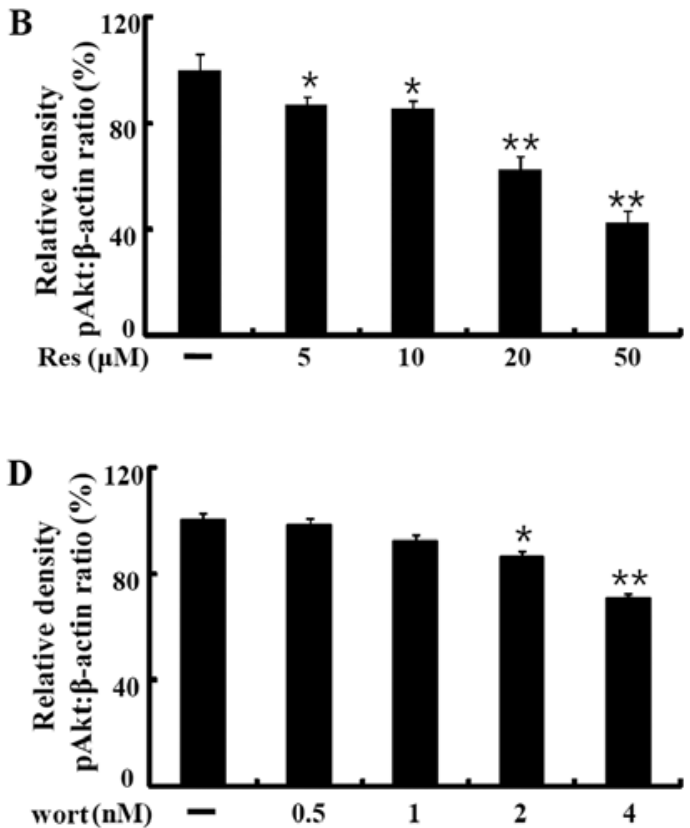

$\mathbf{E}$

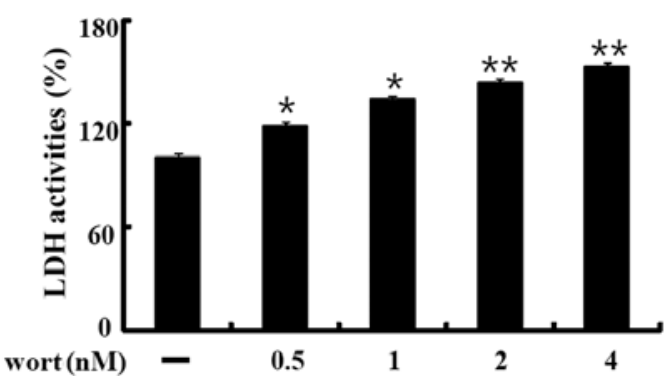

Figure 3. Resveratrol induces cell death through inhibition of Akt phosphorylation. (A and B) HaCaT keratinocytes were incubated with the indicated concentrations of resveratrol $(5,10,20$ and $50 \mu \mathrm{M})$ for $12 \mathrm{~h}$. The treated cells were assessed for phospho-Akt by western blot analysis. (C and D) HaCaT keratinocytes were incubated with wortmannin, an Akt inhibitor, for $12 \mathrm{~h}$ at increasing concentrations (5, 10, 20 and $50 \mu \mathrm{M})$. Western blot analyses for phospho-Akt were performed on HaCaT keratinocytes. $\beta$-actin was used as the loading control. (E) LDH assay was used to quantify LDH release into the medium. Bar graphs indicates the mean $\pm \operatorname{SEM}(\mathrm{n}=3)$. ${ }^{*} \mathrm{p}<0.05,{ }^{* *} \mathrm{p}<0.01$, significant differences between the control and each treatment group. Res, resveratrol; Wort, wortmannin.

and survival signals was investigated by measuring the expression of Akt phosphorylation by western blot analysis. The results showed that resveratrol inhibited Akt activation dose-dependently (Fig. 3A and B). Akt inhibition by exposure to different concentrations of wortmannin $(0.5,1,2$ and $4 \mu \mathrm{M})$ also decreased the phospho-Akt protein levels (Fig. 3C and D). We evaluated the released LDH activity in the media of the $\mathrm{HaCaT}$ keratinocytes treated with wortmannin alone. The data showed that wortmannin dose-dependently increased LDH release (Fig. 3E).

Resveratrol-induced Sirtl increase leads to cell death through Akt phosphorylation. Next, we investigated whether resveratrol-induced Sirt1 activation is involved in Akt phosphorylation. HaCaT keratinocytes were treated with resveratrol with or without sirtinol. Resveratrol decreased phospho-Akt protein expression at $50 \mu \mathrm{M}$, and this effect was restored by the Sirt1 inhibitor, sirtinol (Fig. 4A and B). We next treated the cells with an adenovirus of Sirt1 and found that AKT phosphorylation was decreased dose-dependently. Cells were transfected with adenoviruses expressing Sirtl at a multiplicity of infection
(MOI) of 100, 200, 500 and 1,000 pfu/cell to directly evaluate the role of Sirtl in HaCaT keratinocytes. Sirtl-overexpressing cells exhibited increased Sirtl protein levels and a markedly decrease in acetyl-p53 protein. Overexpression of Sirt1 led to a decrease in phospho-Akt protein levels (Fig. 4C-E). Sirt1, acetyl-p53 and phospho-Akt expression in Ad-LacZ-infected cells was not changed when compared to the levels in the control cells. These results showed that resveratrol-induced Sirtl upregulation led to cell death of HaCaT keratinocytes through phospho-Akt.

\section{Discussion}

Psoriasis is a chronic inflammatory skin disease involving hyperproliferation and abnormal differentiation of epidermal keratinocytes. Several therapies for mild psoriasis are currently available (24). However, due to the complex nature of psoriasis, most therapies have adverse side-effects. Apoptosis is a physiological process in the skin to regulate keratinocyte proliferation and epidermal growth. Lack of apoptosis leads to increase in anti-apoptotic proteins in the skin that cause 
A

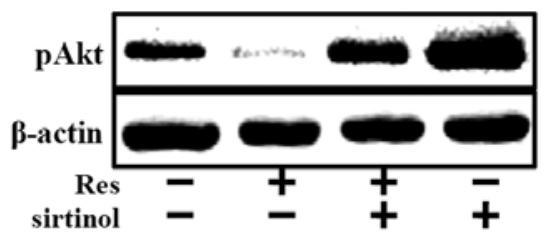

C

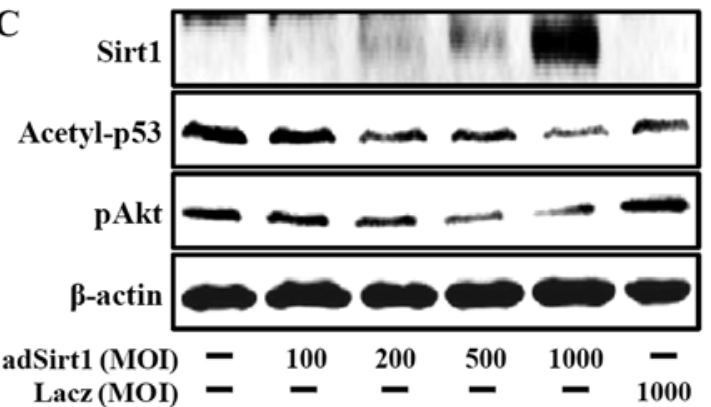

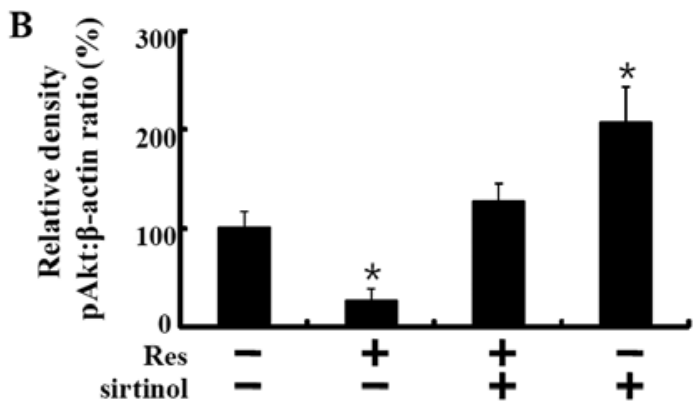

D

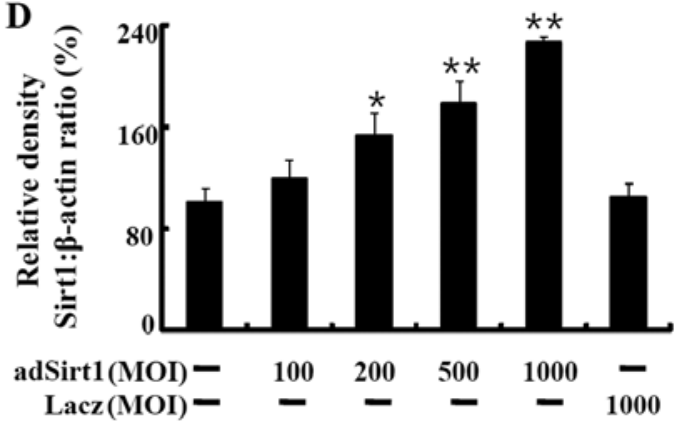

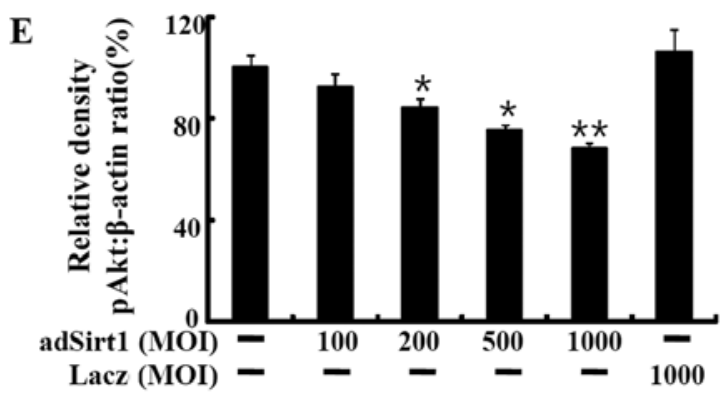

Figure 4. Resveratrol-mediated Sirt1 leads to phospho-Akt inactivation. (A and B) HaCaT keratinocytes were treated with $50 \mu \mathrm{M}$ of resveratrol in the presence of sirtinol $(10 \mu \mathrm{M})$ for $12 \mathrm{~h}$. Phospho-Akt was analyzed by western blotting in the HaCaT keratinocytes. $\beta$-actin was used as the loading control. (C-E) HaCaT keratinocytes were transfected with Sirt1-overexpressing adenovirus (adSirt1) or Lacz-bearing adenovirus (Lacz). Western blotting for Sirt1, acetyl-p53 and phospho-Akt proteins was conducted in the HaCaT keratinocytes. Bar graphs indicate the mean \pm SEM $(n=3) .{ }^{*} p<0.05,{ }^{* *} p<0.01$, significant differences between the control and each treatment group. Res, resveratrol; MOI, multiplicity of infection.

psoriasis (24). We investigated an effective therapy to inhibit hyperproliferation and induce apoptosis in keratinocytes for psoriasis treatment.

Resveratrol (3,5,4'-trihydroxy-trans-stilbene), a natural phenol known as a stilbenoid (25), was found to prevent inflammation and apoptosis of human and murine primary keratinocytes against UVB irradiation or $\mathrm{H}_{2} \mathrm{O}_{2}$ (26-28). However, resveratrol exhibited differential responses in $\mathrm{HaCaT}$ and primary human keratinocytes. Resveratrol sensitized UV-induced apoptosis in $\mathrm{HaCaT}$ keratinocytes (29). Cellular apoptosis is a main mechanism for blocking proliferation of cultured cells, hence we investigated whether resveratrol alone promoted apoptosis in $\mathrm{HaCaT}$ keratinocytes.

Resveratrol has attracted much attention for its ability to enhance the deacetylase activity of Sirt1 (30). Sirt1 is an NAD-dependent histone deacetylase that is a major regulator of multiple biological processes, including the stress response, apoptosis, the regulation of gene transcription and the cell cycle $(12,31)$. Although Sirtl mainly has protective effects against apoptosis in many cells, some cells undergo cell death by Sirt1 expression and activation $(23,32)$. We showed that the resveratrol-mediated increase in Sirtl led to cell death in $\mathrm{HaCaT}$ keratinocytes.

Numerous studies suggest that Akt plays a critical role in tumorigenesis and cell survival. Resveratrol reportedly inhibits skin tumorigenesis through PI3K and Akt, proteins that are implicated in cancer development and progression (33). Akt phosphorylation involved in the apoptotic process raised the possibility that Akt regulates cell survival by directly phosphorylating components of the cell death pathway (21). We found that resveratrol inhibited phospho-Akt and the inhibition of PI3K by wortmannin-mediated resveratrol-induced apoptosis in HaCaT keratinocytes. Our data indicated that resveratrol inhibited cell proliferation and induced apoptosis through the inactivation of Akt signaling. Collectively, the present study demonstrated that resveratrol regulated cell death in HaCaT keratinocytes via Sirt1/phospho-Akt-mediated signaling pathways. Additionally, these findings suggested that resveratrol may be used as a potential therapeutic agent for psoriasis. 


\section{Acknowledgements}

The present study was supported by the Global Ph.D. Fellowship Program through the National Research Foundation of Korea (NRF) funded by the Ministry of Education (2014H1A2A1021117 and 2013R1A1A2063931).

\section{References}

1. Wrone-Smith T, Mitra RS, Thompson CB, Jasty R, Castle VP and Nickoloff BJ: Keratinocytes derived from psoriatic plaques are resistant to apoptosis compared with normal skin. Am J Pathol 151: 1321-1329, 1997.

2. Lebwohl M: Psoriasis. Lancet 361: 1197-1204, 2003.

3. Nickoloff BJ and Nestle FO: Recent insights into the immunopathogenesis of psoriasis provide new therapeutic opportunities. J Clin Invest 113: 1664-1675, 2004.

4. Ortonne N, Ram-Wolff C, Giustiniani J, Marie-Cardine A, Bagot M, Mecheri S and Bensussan A: Human and mouse mast cells express and secrete the GPI-anchored isoform of CD160. J Invest Dermatol 131: 916-924, 2011.

5. Dolinsky VW and Dyck JR: Calorie restriction and resveratrol in cardiovascular health and disease. Biochim Biophys Acta 1812: 1477-1489, 2011.

6. Frémont L: Biological effects of resveratrol. Life Sci 66: 663-673, 2000.

7. Aggarwal BB, Bhardwaj A, Aggarwal RS, Seeram NP Shishodia $S$ and Takada Y: Role of resveratrol in prevention and therapy of cancer: Preclinical and clinical studies. Anticancer Res 24: 2783-2840, 2004.

8. Corpet DE and Pierre F: Point: From animal models to prevention of colon cancer. Systematic review of chemoprevention in min mice and choice of the model system. Cancer Epidemiol Biomarkers Prev 12: 391-400, 2003.

9. Borra MT, Smith BC and Denu JM: Mechanism of human SIRT1 activation by resveratrol. J Biol Chem 280: 17187-17195, 2005.

10. Howitz KT, Bitterman KJ, Cohen HY, Lamming DW, Lavu S, Wood JG, Zipkin RE, Chung P, Kisielewski A, Zhang LL, et al: Small molecule activators of sirtuins extend Saccharomyces cerevisiae lifespan. Nature 425: 191-196, 2003.

11. Hanahan D and Weinberg RA: The hallmarks of cancer. Cell 100: 57-70, 2000.

12. Blander G and Guarente L: The Sir2 family of protein deacetylases. Annu Rev Biochem 73: 417-435, 2004.

13. Luo J, Nikolaev AY, Imai S, Chen D, Su F, Shiloh A, Guarente L and $\mathrm{Gu}$ W: Negative control of p53 by Sir2alpha promotes cell survival under stress. Cell 107: 137-148, 2001.

14. Rajamohan SB,Pillai VB, Gupta M, Sundaresan NR, Birukov KG, Samant S, Hottiger MO and Gupta MP: SIRT1 promotes cell survival under stress by deacetylation-dependent deactivation of poly(ADP-ribose) polymerase 1. Mol Cell Biol 29: 4116-4129, 2009.

15. Yamamori T, DeRicco J, Naqvi A, Hoffman TA, Mattagajasingh I, Kasuno K, Jung SB, Kim CS and Irani K: SIRT1 deacetylates APE1 and regulates cellular base excision repair. Nucleic Acids Res 38: 832-845, 2010.

16. Bai L, Pang WJ, Yang YJ and Yang GS: Modulation of Sirt1 by resveratrol and nicotinamide alters proliferation and differentiation of pig preadipocytes. Mol Cell Biochem 307: 129-140, 2008.
17. Testa JR and Bellacosa A: AKT plays a central role in tumorigenesis. Proc Natl Acad Sci USA 98: 10983-10985, 2001.

18. Kandel ES and Hay N: The regulation and activities of the multifunctional serine/threonine kinase Akt/PKB. Exp Cell Res 253: 210-229, 1999.

19. Brazil DP and Hemmings BA: Ten years of protein kinase B signalling: A hard Akt to follow. Trends Biochem Sci 26: 657-664, 2001

20. Lawlor MA and Alessi DR: PKB/Akt: A key mediator of cell proliferation, survival and insulin responses? J Cell Sci 114: 2903-2910, 2001.

21. Datta SR, Brunet A and Greenberg ME: Cellular survival: A play in three Akts. Genes Dev 13: 2905-2927, 1999.

22. Arboleda MJ, Lyons JF, Kabbinavar FF, Bray MR, Snow BE, Ayala R, Danino M, Karlan BY and Slamon DJ: Overexpression of AKT2/protein kinase Bbeta leads to up-regulation of beta1 integrins, increased invasion, and metastasis of human breast and ovarian cancer cells. Cancer Res 63: 196-206, 2003.

23. Chen S, Xiao X, Feng X, Li W, Zhou N, Zheng L, Sun Y, Zhang Z and Zhu W: Resveratrol induces Sirt1-dependent apoptosis in 3T3-L1 preadipocytes by activating AMPK and suppressing AKT activity and survivin expression. J Nutr Biochem 23: 1100-1112, 2012

24. Zhou LL, Lin ZX, Fung KP, Cheng CH, Che CT, Zhao M, Wu SH and Zuo Z: Celastrol-induced apoptosis in human HaCaT keratinocytes involves the inhibition of NF- $\kappa \mathrm{B}$ activity. Eur $\mathrm{J}$ Pharmacol 670: 399-408, 2011.

25. Soleas GJ, Diamandis EP and Goldberg DM: Wine as a biological fluid: History, production, and role in disease prevention. J Clin Lab Anal 11: 287-313, 1997.

26. Afaq F, Adhami VM and Ahmad N: Prevention of short-term ultraviolet $\mathrm{B}$ radiation-mediated damages by resveratrol in SKH-1 hairless mice. Toxicol Appl Pharmacol 186: 28-37, 2003.

27. Aziz MH, Afaq F and Ahmad N: Prevention of ultraviolet-B radiation damage by resveratrol in mouse skin is mediated via modulation in survivin. Photochem Photobiol 81: 25-31, 2005.

28. Potapovich AI, Kostyuk VA, Kostyuk TV, de Luca C and Korkina LG: Effects of pre- and post-treatment with plant polyphenols on human keratinocyte responses to solar UV. Inflamm Res 62: 773-780, 2013.

29. Vitale N, Kisslinger A, Paladino S, Procaccini C, Matarese G, Pierantoni GM, Mancini FP and Tramontano D: Resveratrol couples apoptosis with autophagy in UVB-irradiated HaCaT cells. PLoS One 8: e80728, 2013.

30. Yu W, Fu YC, Zhou XH, Chen CJ, Wang X, Lin RB and Wang W: Effects of resveratrol on $\mathrm{H}_{2} \mathrm{O}_{2}$-induced apoptosis and expression of SIRTs in H9c2 cells. J Cell Biochem 107: 741-747, 2009.

31. Michan S and Sinclair D: Sirtuins in mammals: Insights into their biological function. Biochem J 404: 1-13, 2007.

32. Chen Q, Ganapathy S, Singh KP, Shankar S and Srivastava RK: Resveratrol induces growth arrest and apoptosis through activation of FOXO transcription factors in prostate cancer cells. PLoS One 5: e15288, 2010.

33. Roy P,Kalra N,Prasad S, George J and Shukla Y: Chemopreventive potential of resveratrol in mouse skin tumors through regulation of mitochondrial and PI3K/AKT signaling pathways. Pharm Res 26: 211-217, 2009 . 